\title{
From effective actions to actual effects in QED*
}

\author{
Holger Gies \\ Institut für theoretische Physik, Universität Tübingen, \\ 72076 Tübingen, Germany
}

November 20, 2018

\begin{abstract}
The construction of low-energy effective actions in QED for several types of external conditions is reviewed. Emphasis is put on the application of these effective actions to a variety of physical effects which represent a manifestation of vacuum polarization. Soft-photon interactions with external electromagnetic fields and/or a heat bath are described, pair production at finite temperature is discussed, and finally a glance at photon-neutrino interactions is provided.
\end{abstract}

\section{Introduction}

The most disturbing ingredient in quantum electrodynamics (QED) is the mass of the electron. It separates the fields and their ubiquitous quantum fluctuations into two classes: those with momenta larger than, and those with momenta smaller than, the electron mass $m$. Naturally, electrons can only appear in the first class which gives rise to the distinctiveness of electromagnetic fields with momenta smaller than $m$. In this talk, we shall turn our attention to these, so-called "soft", fields and their dynamics, and how this dynamics is influenced by the "hard" fluctuations of electrons and photons. Since $m \hat{=} 7.6 \times$ $10^{11} \mathrm{GHz}$, the terminus "soft field" covers a considerably wide range of electromagnetic fields.

From the full quantum theory of electrons and photons, we can arrive at an "effective" description of the soft electromagnetic fields in terms of an effective action; the latter is obtained by averaging over the hard fluctuations and condensing their influence into a number of interaction couplings between the soft fields. This program can also be carried out in the presence of various external perturbations, a so-called modified vacuum, which

${ }^{*}$ Presented at QED 2000, the $2^{\text {nd }}$ Workshop on "Frontier TESTS OF QUANTUM ELECTRODYNAMiCS AND PHYSICS OF THE VACUUM", at Trieste, Italy, Oct. 5-11, 2000 
(a)

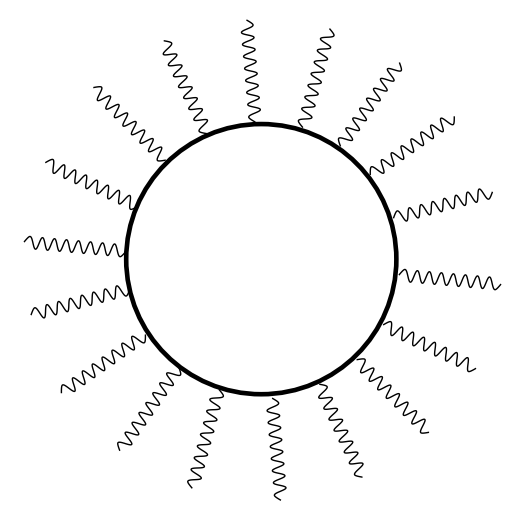

(b)

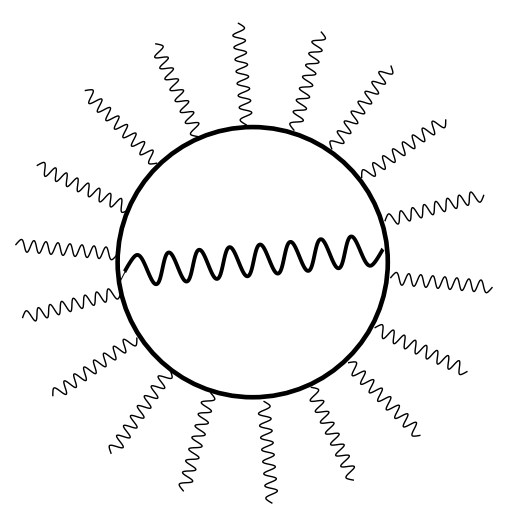

Figure 1: (a) Diagrammatic representation of the QED effective action at the one-loop level corresponding to Eq. (囵). (b) Two-loop level of the effective action as employed in Sect. 2 involving a thermalized radiative photon.

affect the high-momentum fluctuations. After the averaging, the external perturbations are also reflected in the soft-field couplings and thereby modify the dynamics of the soft fields indirectly.

This talk reviews a personal selection of such effective actions adapted to different physical systems and presents some of their applications to physical effects.

Let us begin with a brief sketch of the classic example: the Heisenberg-Euler action [2]. Here, the physical system is defined simply by soft electromagnetic fields being placed in the vacuum. The vacuum, however, is filled with fluctuating electrons and positrons that couple directly to the external fields. Averaging over the fermions results in an interacting theory of the soft fields themselves:

$$
\begin{aligned}
\Gamma_{\mathrm{eff}}^{\mathrm{HE}}= & \int d^{4} x\left\{-\mathcal{F}+\frac{1}{8 \pi^{2}} \int_{0}^{\infty} \frac{d s}{s^{3}} \mathrm{e}^{-\mathrm{i} m^{2} s}\right. \\
& \left.\times\left\{(e s)^{2}|\mathcal{G}| \cot \left[e s\left(\sqrt{\mathcal{F}^{2}+\mathcal{G}^{2}}+\mathcal{F}\right)^{\frac{1}{2}}\right] \operatorname{coth}\left[e s\left(\sqrt{\mathcal{F}^{2}+\mathcal{G}^{2}}-\mathcal{F}\right)^{\frac{1}{2}}\right]+\frac{2}{3}(e s)^{2} \mathcal{F}-1\right\}\right\} \\
= & \int d^{4} x\left(-\mathcal{F}+\frac{8}{45} \frac{\alpha^{2}}{m^{4}} \mathcal{F}^{2}+\frac{14}{45} \frac{\alpha^{2}}{m^{4}} \mathcal{G}^{2}+\mathcal{O}\left(F^{6}\right)\right),
\end{aligned}
$$

where we introduced the only gauge and Lorentz invariants of the electromagnetic field

$$
\mathcal{F}:=\frac{1}{4} F_{\mu \nu} F^{\mu \nu}=\frac{1}{2}\left(\mathbf{B}^{2}-\mathbf{E}^{2}\right), \quad \mathcal{G}:=\frac{1}{4} F_{\mu \nu}{ }^{\star} F^{\mu \nu}=-\mathbf{E} \cdot \mathbf{B} .
$$

Equation (四) displays the one-loop Heisenberg-Euler action in proper-time representation as derived by Schwinger [3] (see Fig. 1(a)); the last line is a weak-field expansion thereof including the 4-photon-vertex level. The first term is the classical Maxwell term and the interaction terms $\sim \frac{\alpha^{2}}{m^{4}}$ are given in one-loop approximation. Only because the electron is

\footnotetext{
${ }^{1}$ For technical details about the construction of these actions, the interested reader is referred to the original literature; for a comprehensive collection of large parts of the material presented here, see [1].
} 
(a)

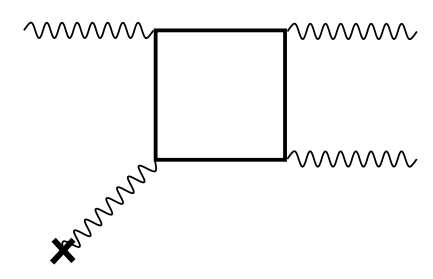

(b)

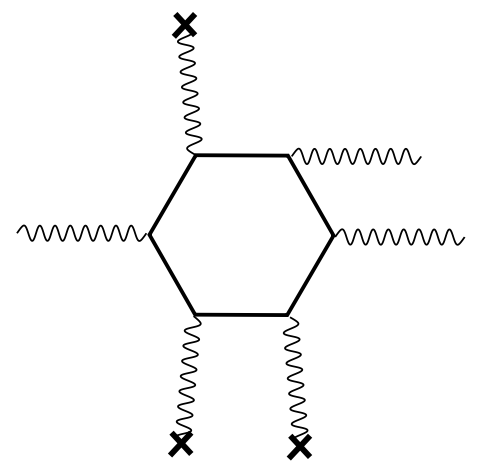

Figure 2: (a) The photon-splitting box graph is suppressed at zero temperature, but becomes dominant at finite temperature (cf. Eq. (15)). (b) The hexagon graph dominates zero-temperature photon splitting (cf. Eq. (田)). Crosses denote couplings to the magnetic background field.

so "heavy", can the world of human experience be appropriately approximated by what we call classical electrodynamics owing to the smallness of the next-to-leading order terms.

Beyond the classical approximation, a number of effects appear as a consequence of the self-interactions of the soft fields. In the first place, we have light-by-light scattering as a direct violation of the classical superposition principle. Of course, the integrated total elastic cross section as derived from the last line of Eq. (11) is very small:

$$
\sigma=\frac{973}{10125} \frac{\alpha^{2}}{\pi} r_{0}^{2}\left(\frac{\omega}{m}\right)^{6}
$$

where $r_{0}$ is the classical electron radius, and the center-of-mass frequency of the photon is small by assumption, $\omega \ll m$.

As a second example, we may consider two different types of soft fields, e.g., a propagating soft photon in the background of an electromagnetic field. As was shown by Adler [4, such a photon can decay into two photons in the presence of a background magnetic field. This photon splitting can act as a production mechanism of polarized light near strongly magnetized astrophysical objects because the splitting of photons with perpendicular polarization WRT the $\mathbf{B}$ field into parallelly polarized ones is preferred, $\left(\perp \rightarrow\left\|_{1}+\right\|_{2}\right)$. The absorption coefficient of this process as derived from Eq. (1) is given by

$$
\kappa \simeq 0.116 \mathrm{~cm}^{-1}\left(\frac{e B}{m^{2}}\right)^{6} \sin ^{6} \theta_{B}\left(\frac{\omega}{m}\right)^{5}, \quad B \ll B_{\mathrm{cr}}:=\frac{m^{2}}{e}, \quad \theta_{B}=\Varangle \text { (prop. direct., B). }
$$

One peculiarity of this process is the $B^{6}$ dependence: it indicates that three couplings of the background field to the $e^{+} e^{-}$loop are necessary for the lowest-order process. Together with the one incoming and the two outgoing photons, this constitutes a hexagon graph; the box graph with one coupling to the $B$ field is suppressed owing to properties of the Lorentz algebra of the field strength tensor (see Fig. 2).

The third example is the seeming paradox that light in an electromagnetic background no longer propagates "at the speed of light". The vacuum modified by the background field acts like a medium in classical electrodynamics shifting the phase and group velocities 
of propagating soft photons, including effects of birefringencef] and dispersion. In many cases, the polarization-averaged deformation of the light cone $k^{2}$ can be described by [6]

$$
k^{2}=Q\left\langle T^{\mu \nu}\right\rangle k_{\mu} k_{\nu}
$$

where $\left\langle T^{\mu \nu}\right\rangle$ denotes the expectation value of the energy-momentum tensor in the modified vacuum, and the proportionality factor $Q$ depends on the parameters of the effective action (recently, a formulation of Eq. (5) in terms of an "effective metric" has been given [7]). For the case of a magnetic background field, the classical result as obtained from the last line of Eq. (1) for the velocity of light is [4, 8, 9]:

$$
v \simeq 1-\frac{11}{45} \frac{\alpha^{2}}{m^{4}} B^{2} \sin ^{2} \theta_{B}
$$

As a final introductory example, we would like to mention the Schwinger mechanism for $e^{+} e^{-}$pair production in electric fields, representing the instability of the vacuum that is modified by an electric field. The pair production rate per unit volume and time is related to the imaginary part of the effective action (11):

$$
w=2 \operatorname{Im} \mathcal{L}_{\mathrm{eff}}^{\mathrm{HE}}=\frac{(e E)^{2}}{4 \pi^{3}} \sum_{n=1}^{\infty} \frac{\exp \left(-\frac{n \pi m^{2}}{e E}\right)}{n^{2}}, \quad \Gamma_{\mathrm{eff}}^{\mathrm{HE}}=\int d^{4} x \mathcal{L}_{\mathrm{eff}}^{\mathrm{HE}} .
$$

It is noteworthy that the Eq. (7) is a nonperturbative result, in the sense that it cannot be obtained from an expansion of the action in terms of the field strength. The Schwinger mechanism has recently been verified in the famous SLAC E144 experiment [10]. Moreover, it has become a building block of various pair-production models for heavy-ion collisions.

Generalizations to the Heisenberg-Euler effective action have been investigated for a variety of further modified vacua including gravitational backgrounds, finite temperature and/or density, nontrivial boundary conditions (Casimir vacua), and even couplings to the QCD vacuum. Exemplarily, we consider the presence of a heat bath in the following section. In Sect. 3, we go one step further and enlarge the particle content of our system by taking neutrinos into account.

\section{QED effective action at finite temperature}

The generalization of the precedingly presented philosophy to finite temperature represents a challenge from a purely theoretical as well as a phenomenological viewpoint? Phenomenologically, the presence of a heat bath gives rise to thermal fluctuations of the

\footnotetext{
${ }^{2}$ The world is currently awaiting the observation of the birefringence phenomenon as a first direct verification of the effective nonlinearity of electrodynamics [5].

${ }^{3}$ In the literature, this problem has been a touchstone for the various finite-temperature formalisms; after decades of discussion, real-time formalism [1] and imaginary-time formalism [12] have come to congruent results.
} 
electrons, positrons and photons in the vacuum. Contrary to their quantum analogue, these fluctuations are strictly on-shell (but can couple to off-shell quantum fluctuations) and create a (neutral) plasma, containing thermally excited (charged) particles. The latter can exert an influence on the soft fields under consideration, which renders their dynamics temperature dependent.

Let us first glance over the theoretical aspects. For the formalism, the correspondence between quantum field theory and statistical mechanics for thermal equilibrium is employed, leading to propagators and wave functions that are periodic in imaginary time with period $\beta=1 / T$. Furthermore, Lorentz invariance is broken explicitly, since the rest frame of the heat bath is distinguished; as a new algebraic element, the 4-velocity vector of the heat bath $u^{\mu}$ appears $\left(u^{\mu}=(1,0,0,0)\right.$ in the heat-bath rest frame) and allows for the construction of a further invariant beside the temperature itself:

$$
\mathcal{E}:=\left(u_{\alpha} F^{\alpha \mu}\right)\left(u_{\beta} F^{\beta}{ }_{\mu}\right), \quad \mathcal{E}=\mathbf{E}^{2} \text { in the heat-bath rest frame. }
$$

The invariants $\mathcal{E}, \mathcal{F}, \mathcal{G}$ are complete on a classical level, in the sense that they contain the entire information about the soft fields. On the quantum level, a new aspect appears: since the periodicity condition of the wave functions has to be respected, gauge transformations also have to satisfy this requirement, leading to a restricted class of gauge transformations obeying periodicity in imaginary time. This makes room for another gauge invariant quantity:

$$
\bar{A}_{u}(\mathbf{x})=\frac{1}{\beta} \int_{0}^{\beta} d \tau A_{u}\left(x^{\mu}+\mathrm{i} \tau u^{\mu}\right), \quad A_{u}:=A^{\mu} u_{\mu},
$$

where $\mathbf{x}$ denotes the components of $x^{\mu}$ orthogonal to $u^{\mu}$. The physical interpretation of $\bar{A}_{u}$ is that it has to be identified with a chemical potential: namely, $e \bar{A}_{u}(\mathbf{x})$ denotes the electrostatic energy cost for placing an electron at position $\mathbf{x}$. The gauge field $A_{\mu}$ therefore contains considerably more information about the system at finite than at zero temperature. Moreover, the restricted class of gauge transformations no longer protects the zero-mass of the photon as stringently as at zero temperature; it is exactly the $\bar{A}_{u}$ part of the gauge field that acquires a mass by quantum fluctuations which has to be identified with the Debye screening mass (see below).

Also, the mechanism by which the quantum invariant $\bar{A}_{u}$ enters the effective action is interesting: the periodicity condition for the quantum fields leads to a compactification of the spacetime manifold in imaginary-time direction - the manifold becomes cylindrical, $\mathbb{R}^{3} \times S^{1}$. The virtual $e^{+} e^{-}$loops therefore fall into different homotopy classes characterized by their number of windings around the cylinder. This winding number is measured with the aid of $\bar{A}_{u}$, so that the $\bar{A}_{u}$ dependence of the final effective action is of topological origin.

Let us now come to the results for the thermal effective action; at the one-loop level various exact representations are known for arbitrary values of temperature and field strength [11, 12, 13]. Here, we confine ourselves to some limiting cases. Naturally, the electron mass scale distinguishes between a high-, an intermediate- and a low-temperature domain, $T \gg m, T \sim m, T \ll m$. 
As a first limit, we consider $T \gg m$ and vanishing background field strengths, so that the one-loop thermal contribution $\Gamma_{\text {eff }}^{1 T}$ only depends on $\bar{A}(u)$ and can be expanded as $\Gamma_{\text {eff }}^{1 T}=\int d^{4} x\left(-\frac{1}{2} \partial_{\mu} \bar{A}_{u} \partial^{\mu} \bar{A}_{u}+\frac{m_{\text {eff }}^{2}}{2}\left(\bar{A}_{u}\right)^{2}+\mathcal{O}\left(\bar{A}_{u}^{4}\right)\right)$. Here, we can read off the effective mass that determines the Debye screening effect of electric fields:

$$
m_{\text {eff }}^{2}(T)=\frac{(e T)^{2}}{3}, \quad T \gg m,
$$

which is the well-known result found in the literature employing different methods.

Particularly interesting is the limiting case of soft background fields at high temperature $T \gg m$ (setting $\bar{A}_{u}=0$ for simplicity). At one-loop order, the thermally fluctuating electrons move ultra-relativistically so that the scale of the loop process is no longer set by the electron mass $m$, but by the temperature $T$. Therefore, in the limit $T \rightarrow \infty$, the loop particles become infinitely heavy and decouple from the theory. Consequently, vacuum polarization and thereby any nonlinear interaction of the soft fields is strongly suppressed. The remaining linear terms are

$$
\Gamma_{\text {eff }} \equiv \Gamma_{\text {eff }}^{\mathrm{HE}}+\Gamma_{\text {eff }}^{1 T}=\int d^{4} x\left(-\mathcal{F}-\frac{2 \alpha}{3 \pi} \mathcal{F} \ln \frac{T}{m}+\frac{\alpha}{6 \pi} \mathcal{E}\right), \quad T \gg m .
$$

Besides the classical Maxwell term, we find a finite logarithmic renormalization of the charge running with temperature; this term modifies, e.g., the Compton amplitude. The last term $\sim \mathcal{E}$ is responsible for a decelerated light propagation (see below).

In the opposite limit of low temperature, the thermal one-loop contribution to be added to Eq. (1) reads in a weak-field approximation (at zero chemical potential) 13:

$$
\begin{aligned}
\Gamma_{\text {eff }}^{1 T} \simeq \int d^{4} x & \left\{\frac{\alpha}{\pi}\left[\frac{1}{6} \sqrt{\frac{2 \pi m}{T}} \mathcal{E}+\frac{2}{3} \sqrt{\frac{2 \pi T}{m}} \mathcal{F}\right] \mathrm{e}^{-m / T}\right. \\
-\alpha^{2}\left[\frac{1}{36}\right. & \frac{m^{4}}{T^{4}} \sqrt{\frac{\pi T}{2 m}} \mathcal{E}^{2}-\frac{\pi}{90} \frac{m^{3}}{T^{3}}\left(8 \mathcal{F} \mathcal{E}+\mathcal{G}^{2}\right)+\frac{1}{45} \frac{m^{2}}{T^{2}}\left(8 \mathcal{F}^{2}-8 \mathcal{F} \mathcal{E}+13 \mathcal{G}^{2}\right) \\
+ & \left.\left.\frac{\pi}{30} \frac{m}{T}\left(4 \mathcal{F}^{2}+7 \mathcal{G}^{2}\right)+\frac{4}{45}\left(4 \mathcal{F}^{2}+7 \mathcal{G}^{2}\right)\right] \frac{\mathrm{e}^{-m / T}}{m^{4}}\right\}, \quad T \ll m .
\end{aligned}
$$

Equation (12) can serve as a starting point for similar explorations of physical effects as discussed above for $\Gamma_{\text {eff }}^{\mathrm{HE}}$ at $T=0$; however, we observe that the thermal one-loop effective action at low temperature $T \ll m$ is exponentially suppressed by the electron mass; consequently, any actual effect will be immeasurably small in this limit. The physical reason for this suppression is obvious: since the electron spectrum exhibits a mass gap $m$, a heat bath of temperature $T \ll m$ can hardly excite electronic states to a significant amount.

This situation changes drastically when we take a look at the next order in perturbation theory, the two-loop level. Here, an additional radiative photon has to be considered within the loop. But the photon is massless, so that higher photon states can be thermally excited 
even at small values of temperature. This is the reason why we have to expect that the thermal two-loop contribution to the effective action (see Fig. 1(b)) exceeds the thermal one-loop part in the low-temperature limit. This two-loop dominance connotes an inversion of the usual loop hierarchy and signifies that the one-loop approximation represents an inconsistent truncation of the theory in this temperature domain; herein, at least one radiative photon has to be taken into account for correct results.

This situation even intensifies for the imaginary part of the effective action which is related to pair production in electric fieldst. By construction, the thermal one-loop contribution is purely real (this is seen directly in the real-time formalism [11]). Since the one-loop approximation deals only with thermalized $e^{+}$and $e^{-}$which are on-shell by construction, there can be no thermal contribution to the pair-production rate to this order of calculation; this is because the $e^{+} e^{-}$pair has to go off-shell in order to "tunnel" through the mass gap $2 m$. Again at two loop, the situation is different, because then, the thermalized on-shell radiative photon can couple to the fermionic off-shell quantum fluctuations; in this case, a thermal contribution to pair-production is no longer prohibited.

These arguments make clear that an understanding of thermal physics of soft electromagnetic fields requires a two-loop calculation. The cumbersome calculation is simplified by the fact that only the internal radiative photon has to be thermalized because thermal fermion contributions are suppressed by the electron mass for $T \ll m$. The thermal twoloop contributions finally read in a weak-field approximation (at zero chemical potential) 14]:

$$
\begin{aligned}
\Gamma_{\mathrm{eff}}^{2 T}= & \int d^{4} x\left\{\frac{44 \alpha^{2} \pi^{2}}{2025} \frac{T^{4}}{m^{4}}(\mathcal{F}+\mathcal{E})-\frac{2^{6} \cdot 37 \alpha^{3} \pi^{3}}{3^{4} \cdot 5^{2} \cdot 7} \frac{T^{4}}{m^{4}} \frac{\mathcal{F}(\mathcal{F}+\mathcal{E})}{m^{4}}\right. \\
& \left.+\frac{2^{13} \alpha^{3} \pi^{5}}{3^{6} \cdot 5 \cdot 7^{2}} \frac{T^{6}}{m^{6}}\left(2 \mathcal{F}^{2}+6 \mathcal{E} \mathcal{F}+3 \mathcal{E}^{2}-\mathcal{G}^{2}\right) \frac{1}{m^{4}}+\mathcal{O}\left(F^{6},(T / m)^{8}\right)\right\}, \quad T \ll m
\end{aligned}
$$

We observe a power-law dependence on the temperature starting with $T^{4}$; therefore, the two-loop contribution indeed always wins out over the one-loop term for sufficiently low $T$. This completes our primary picture of the thermal part of the effective action of QED: at small values of $T$, the two-loop part governs the dynamics of soft fields; numerically, we find two-loop dominance for $T / m \lesssim 0.05$. Above, the one-loop part reseizes power over the soft fields in the intermediate-temperature domain $T \sim m$ and beyond. Only the thermal imaginary part is completely controlled by the two-loop action.

Now let us come to the physics contained in Eqs. (13), (12) and (11), beginning with the subject of light propagation. The light cone condition for thermal vacua (cf. Eq. (5))

\footnotetext{
${ }^{4}$ Here it is tacitly assumed that the system with electric fields remains close to thermal equilibrium.
} 

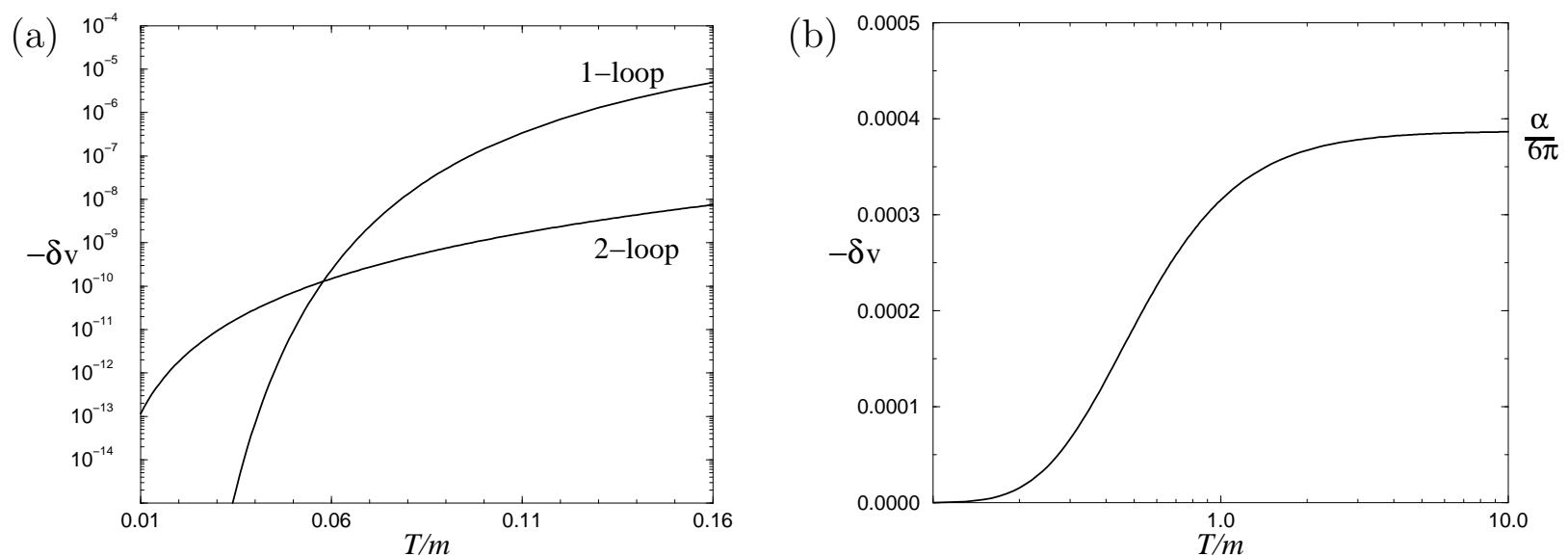

Figure 3: (a) Low-temperature velocity shift $\delta v$ in units of the vacuum velocity $c=1$ versus temperature $T / m$; the one-loop contribution exceeds the two-loop result at $T / m \simeq 5 \%$. (b) Thermally induced velocity shift $-\delta v$ in the intermediate-temperature domain $T \sim m$. The maximum velocity shift $-\delta v=\frac{\alpha}{6 \pi}$ is already approached at comparably low temperatures.

has been derived in [15]; the final results read [16, 15, 14]:

$$
\begin{array}{rlrl}
v_{2 \text {-loop }} & =1-2 \frac{44}{2025} \alpha^{2} \pi^{2} \frac{T^{4}}{m^{4}}+\mathcal{O}\left(T^{8} / m^{8}\right), \quad T \ll m, \\
v_{\text {1-loop }} \simeq 1-\frac{\alpha}{6} \sqrt{\frac{2}{\pi}} \sqrt{\frac{m}{T}} \mathrm{e}^{-\frac{m}{T}}, & T \lesssim m, & \\
v_{\text {1-loop }}=1-\frac{\alpha}{6 \pi}+\mathcal{O}\left(m^{2} / T^{2}\right), & T \gtrsim m .
\end{array}
$$

We observe that the phase and group velocities of light decrease for increasing $T$ until the velocity shift reach a maximum of $\delta v=\alpha /(6 \pi)$ at $T$ a bit above $m$ (see Fig. 3). For $T / m \gtrsim 6$, the considerations become meaningless, because the thermal plasma develops a plasma frequency corresponding to the Debye screening mass that acts as a cutoff for the low-frequency modes. For these values of $T$, "soft photons" simply no longer exist.

Obviously, these velocity shifts are extremely difficult to measure; perhaps one can make use of the fact that certain hot regions in the universe such as stellar atmospheres or nebular structures can act as lenses. Beyond that, it is interesting to note that, e.g., even in the standard model of cosmology, the velocity of light has not been constant during the evolution of the universe. Incidentally, velocity shifts induced by finite $T$ and a magnetic field have also been calculated; unfortunately, the combination of both vacuum modifications leads rather to a washout of the single effects than to an amplification.

Next, we turn to thermally induced photon splitting [13, 14], motivated by the fact that strong magnetic fields and finite temperature (and density) may be encountered near compact astrophysical objects. The new feature of the thermal process is that the box graph with one coupling to the background field (see Fig. 2(a)) is no longer suppressed by Lorentz symmetry, since the latter is broken by the presence of the heat bath; it is indeed the new invariant $\mathcal{E}$ which is responsible for the lowest-order contribution to the thermal 
absorption coefficient of the splitting process $\left(\perp \rightarrow\left\|_{1}+\right\|_{2}\right)$ :

$$
\frac{\kappa^{T}}{m}=\frac{1}{2^{6} \cdot 3 \cdot 5 \pi^{2}}\left(\frac{e B}{m^{2}}\right)^{2} \sin ^{2} \theta_{B}\left(\frac{\omega}{m}\right)^{5}\left(\partial_{\mathcal{E} \mathcal{F}} \mathcal{L}\right)^{2} m^{8}
$$

where $\partial_{\mathcal{E} \mathcal{F}} \mathcal{L}$ denotes the coefficients of those terms in Eqs. (13) and (12) which are bilinear in $\mathcal{E}$ and $\mathcal{F}$. Note the different dependence on $B$ arising from the box graph contrary to Eq. (4).

Soft photons with frequencies below the pair-production threshold are also exposed to further absorption processes: first, the photons can directly scatter with the $e^{+} e^{-}$plasma; the absorption coefficient for this Compton process can be estimated to give [13]

$$
\frac{\kappa_{\mathrm{C}}}{m} \simeq \frac{8 \alpha^{2}}{3 \pi m^{3}} \int_{0}^{\infty} d p \frac{p^{2}}{\mathrm{e}^{\omega_{e} / T}+1}
$$

where $\omega_{e}$ denotes the fermion energy $\omega_{e}=\sqrt{p^{2}+m^{2}}$. The second competing process is the scattering between the propagating photon and the blackbody radiation of the thermal heat bath, i.e., light-by-light scattering as discussed above. This absorption coefficient reads 14 :

$$
\frac{\kappa_{\gamma \gamma}}{m}=\frac{7 \cdot 139}{2^{5} \cdot 3^{7} \cdot 5^{6}} \frac{\pi^{9}}{\zeta(3)^{2}} \alpha^{4}\left(\frac{T}{m}\right)^{6}\left(\frac{\omega}{m}\right)^{3} \simeq 5.21 \cdot 10^{-11}\left(\frac{T}{m}\right)^{6}\left(\frac{\omega}{m}\right)^{3}, \quad T \ll m .
$$

These absorption coefficients have been depicted in Fig. 4 for typical values of the parameters. The two-loop contribution dominates over the one-loop contribution for $T / m \leq 0.041$. However, Compton scattering is the dominant absorption in the intermediate-temperature domain, $T \sim m$, and above; whereas $\gamma \gamma$ scattering with the heat-bath photons can become the most important process in the low-temperature domain for weak fields. The latter processes do not contribute to the generation of polarized light, but rather wash out the anisotropies stemming from photon splitting; therefore, the actual value of the temperature or magnetic field of a pulsar might be inferred from a measurement of the anisotropies. Since the photon-splitting amplitudes have to be added coherently, a hypersurface exists in parameter space where the amplitudes interfere totally destructively. In actual measurements, this effect might be visible as a sharp inverse peak in a plot of the polarization asymmetries.

Finally, we turn to thermal contributions to the Schwinger mechanism of pair production in electric fields. Combining the classical zero-temperature result [3] with the leading thermal two-loop contribution [14], we find in the low-temperature domain, $T \ll m$ :

$$
\begin{aligned}
& \operatorname{Im} \mathcal{L}_{\text {eff }}\left(e E \ll m^{2}\right)=m^{4} \mathrm{e}^{-\pi / \eta}\left(\frac{\eta^{2}}{8 \pi^{3}}+\frac{\alpha \pi^{2}}{180} \frac{1}{\eta^{2}} \frac{T^{4}}{m^{4}}\right) \simeq m^{4} \mathrm{e}^{-\pi / \eta}\left(4 \cdot 10^{-3} \eta^{2}+4 \cdot 10^{-4} \frac{T^{4} / m^{4}}{\eta^{2}}\right) \\
& \operatorname{Im} \mathcal{L}_{\text {eff }}\left(e E \gg m^{2}\right)=m^{4} \eta\left(\frac{\eta}{48 \pi}+\frac{\alpha \pi}{270} \frac{T^{4}}{m^{4}}\right) \simeq m^{4} \eta\left(6.6 \cdot 10^{-3} \eta+8.5 \cdot 10^{-5} \frac{T^{4}}{m^{4}}\right),
\end{aligned}
$$


(a): $\quad \frac{e B}{m^{2}}=0.2, \frac{\omega}{m}=1=\sin \theta_{B}$

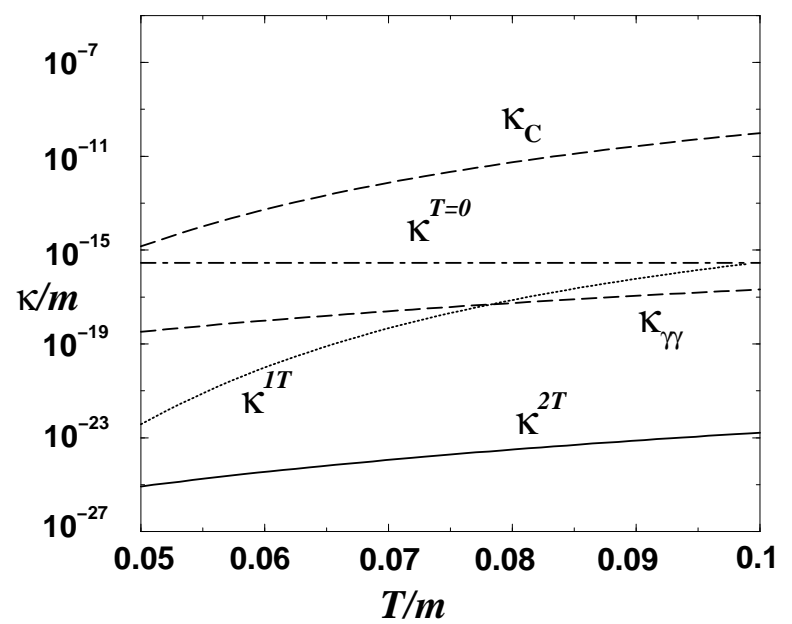

(b): $\quad \frac{e B}{m^{2}}=10^{-4}, \frac{\omega}{m}=1=\sin \theta_{B}$

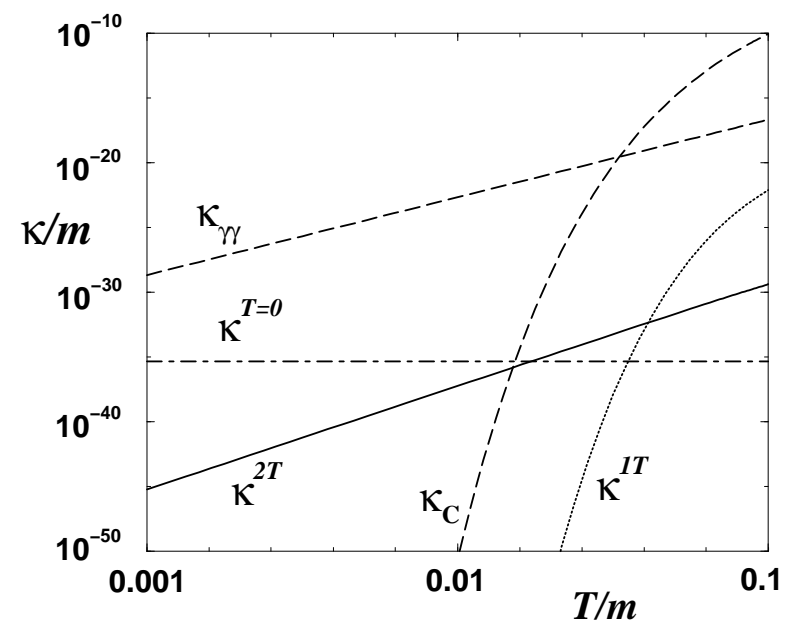

Figure 4: Absorption coefficient $\kappa$ in units of the electron mass versus temperature $T$ in units of the electron mass. In Fig. (a), the various contributions are plotted for parameter values of a realistic astrophysical system. In Fig. (b), the parameters are chosen in such a way that the two-loop dominance over the one-loop and the Compton process is revealed; the photon-photon scattering contribution cannot be surpassed in the low-temperature limit.

where we abbreviated $\eta=\frac{e E}{m^{2}}$. These equations show that the low-temperature contribution to $\operatorname{Im} \mathcal{L}_{\text {eff }}$ can be neglected for strong electric fields; the physical reason for this lies in the fact that the nonlinearities of pure (zero- $T$ ) vacuum polarization exceed the polarizability of the thermally induced real plasma by far in the strong field limit. By contrast, in the limit of weak electric fields, thermal effects can increase the pair-production probability significantly. Of course, for these values of $\eta$, the total imaginary part is very small due to the inverse power of $\eta$ in the exponential.

\section{Effective action for photon-neutrino interactions}

As mentioned above, if the electron had a much lighter sister, we would experience our world quite differently, because (classical) electrodynamics would be inherently nonlinear. In fact, there are very light fermions, the neutrinos, but, (un-)fortunately, they do not couple to electromagnetic fields in a sufficiently strong way. But they nevertheless couple to these fields via intermediary electrons and gauge bosons. Therefore, if we are interested in the physics below the electron mass scale, neutrinos have to be taken into consideration in addition to soft electromagnetic fields. In particular, we are looking for an effective action governing the dynamics of soft photons interacting with neutrinos.

Since we only want to consider energies far below the heavy gauge boson masses, we may start from another effective theory, the Fermi theory, describing the four-fermion 
interaction between neutrinos and the leptons?. For soft photons and "soft" neutrinos $\nu$, we arrive at their effective theory by integrating out (averaging over) the actual "heaviest" particle, i.e., the electron, and find

$$
\Gamma_{\mathrm{eff}}=\frac{G_{\mathrm{F}}}{\sqrt{2}} \frac{1}{e} \int d^{4} x \bar{\nu} \gamma^{\mu}\left(1+\gamma_{5}\right) \nu\left(g_{\mathrm{V}}\left\langle j^{\mu}\right\rangle^{A}+g_{\mathrm{A}}\left\langle j_{5}^{\mu}\right\rangle^{A}\right)
$$

where we introduced the expectation values of the electronic vector and axialvector current in a background field $A_{\mu}$. Diagrammatically, Eq. (19) describes an electron loop in a background field with a $\nu \bar{\nu}$ insertion as depicted in Fig. 5. $G_{\mathrm{F}}$ denotes the Fermi constant.

The vector current expectation value can easily be obtained using the well-known Heisenberg-Euler action (11) and the formula $\left\langle j_{\mu}\right\rangle^{A}=-\delta \Gamma_{\text {eff }}^{H E} / \delta A^{\mu}$. In this way, one obtains a derivative expansion of the vector current around a strong field. For example, the term which is third order in the field and first order in derivatives was used by Dicus and Repko [17] in their study of $\nu \gamma \rightarrow \nu \gamma \gamma$ and cross processes (see also [18]), and by Shaisultanov [19] for investigating the $\nu \gamma \rightarrow \nu \gamma$ and crossed processes in a background magnetic field. Unfortunately, such a simple formula for the axialvector current $\left\langle j_{5}^{\mu}\right\rangle$ does not exist, so it must be calculated from first principles. This has been achieved recently in [20] for arbitrarily strong electromagnetic background fields in a first-order gradient expansion. The necessary basic equation is the relation between the axialvector current and the axialvector-vector amplitude, i.e., the axial analogue of the polarization tensor,

$$
\left\langle j_{5}^{\mu}\right\rangle^{A}=\left.\frac{1}{3} \partial_{\sigma} F_{\alpha \beta} \frac{\partial^{2}}{\partial k^{\sigma} \partial k^{\alpha}}\left[\Pi_{5}^{\beta \mu}(-k)\right]\right|_{k=0},
$$

which holds to first order in derivatives. . The axialvector-vector amplitude has been calculated independently and with very different methods in [21] and [22]. Here, we shall be satisfied by citing the final result for the axialvector current in weak-field expansion:

$$
\begin{aligned}
&\left\langle j_{5}^{\mu}\right\rangle^{A}= \frac{e^{3}}{24 \pi^{2} m^{2}}\left(\partial^{\mu} \mathcal{G}+\left(\partial^{\alpha} F_{\alpha \beta}\right) F^{* \beta \mu}\right) \\
&+\frac{e^{5}}{90 \pi^{2} m^{6}} \partial_{\sigma} F_{\alpha \beta}\left[\mathcal{G}\left(F^{\beta \alpha} g^{\mu \sigma}+F^{\beta \sigma} g^{\mu \alpha}\right)+\left(F^{* \beta \alpha}\left(F^{2}\right)^{\mu \sigma}+F^{* \beta \sigma}\left(F^{2}\right)^{\mu \alpha}\right)\right. \\
&\left.\quad-\mathcal{F}\left(3 F^{* \mu \beta} g^{\alpha \sigma}+F^{* \beta \alpha} g^{\mu \sigma}+F^{* \beta \sigma} g^{\mu \alpha}\right)\right] .
\end{aligned}
$$

Incidentally, the first term can also be obtained from the famous triangle diagram [23]. Upon insertion of Eq. (21) and the well-known vector current into Eq. (19), we arrive at the analogue of the Heisenberg-Euler action for the case involving an axialvector coupling. This provides us with the effective action for neutrino interactions with an arbitrary soft electromagnetic field.

\footnotetext{
${ }^{5}$ In the following, we only consider the influence of electrons; the heavier mass of the other leptons (and quarks) strongly suppresses their additional effects.

${ }^{6} \mathrm{~A}$ similar equation holds also for the vector current and the polarization tensor.
} 


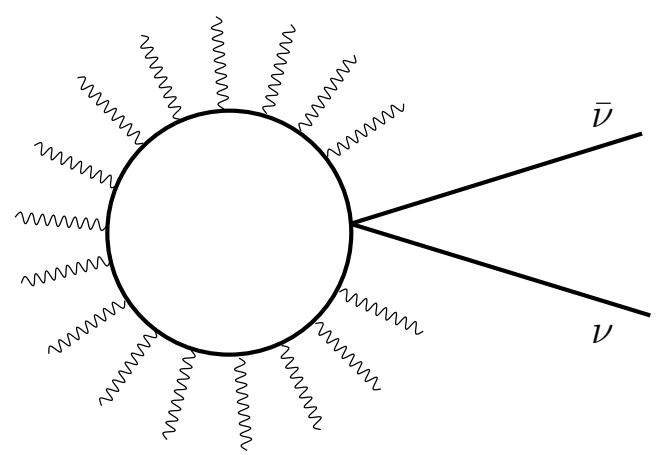

Figure 5: Diagrammatic representation of the effective action for soft electromagnetic fields coupling to neutrinos.

The application of this effective action to real physical systems has not been fully explored up to now, and we shall sketch only a few examples, concentrating on the axialvectorcoupling sector. The first example resembles to some degree the photon-splitting process: Cherenkov radiation of neutrinos in magnetic fields. Since the velocity of light is reduced in magnetic fields (cf. Eq. (6)), neutrinos can propagate "faster than light" and emit Cherenkov radiation. For massless neutrinos propagating perpendicular to the magnetic field, the rate for producing collinear Cherenkov radiation with $\|$ polarization WRT the magnetic field is 24]

$$
\Gamma_{\nu \rightarrow \nu \gamma}\left(m_{\nu}=0\right)=\frac{16 \alpha G_{\mathrm{F}}^{2}}{3^{3} \cdot 5^{3} \cdot(4 \pi)^{4}} E^{5}\left(\frac{B}{B_{\mathrm{cr}}}\right)^{6}, \quad B \ll B_{\mathrm{cr}},
$$

where $E$ is the incident neutrino energy. The result is independent of neutrino flavors. Similarly to the case of photon splitting (at $T=0$ ), we observe a $B^{6}$ dependence signifying three couplings of the background field to the electron loop as the lowest-order contribution; together with the neutrino vertex and the outgoing photon, this corresponds to a pentagon diagram, represented by the last two lines in Eq. (21). The triangle diagram is suppressed for symmetry reasons, similarly to the box diagram in photon splitting.

This suppression no longer holds if we take nonvanishing neutrino masses $m_{\nu}$ into account. Then the dominant mass-dependent contribution arises from the first line of Eq. (21), yielding 23]

$$
\begin{aligned}
& \Gamma_{\nu \rightarrow \nu \gamma}\left(m_{\nu} \neq 0\right)= \frac{7}{2^{9} \cdot 3^{4} \cdot 5^{2}} \frac{\alpha^{2}}{\pi^{4}} m\left(G_{\mathrm{F}} m^{2}\right)^{2}\left(\frac{E}{m}\right)^{3}\left(1-\frac{E_{\mathrm{min}}^{2}}{E^{2}}\right)^{5}\left(\frac{m_{\nu}}{m}\right)^{2}\left(\frac{B}{B_{\mathrm{cr}}}\right)^{4} \theta\left(E-E_{\mathrm{min}}\right), \\
& \text { where } \quad E_{\min }:=\sqrt{\frac{45 \pi}{7 \alpha}} m_{\nu} \frac{B_{\mathrm{cr}}}{B} .
\end{aligned}
$$

The existence of such a mass-dependent threshold energy $E_{\min }$ for the incoming neutrino arises from the Cherenkov condition: the neutrino must move "faster than light" in the $B$-field background. Astonishingly, the transition rate (23) never wins out over the massindependent contribution of Eq. (22). This nontrivial zero-result should serve as a counterexample for the general belief that non-zero neutrino masses always open a window in parameter space for new effects. 
As as second example, let us consider $\bar{\nu} \nu$ pair emission in varying electromagnetic fields 23]. The pure QED-analogue of $e^{+} e^{-}$pair emission in such fields, though it is textbook knowledge, remains phenomenologically unimportant because of the "heavy" mass of the electron. The similar mechanism for neutrinos benefits from the smallness of the neutrino mass, and originates from the first term of Eq. (21) as a part of the triangle diagram.

This term gives rise to the matrix element $\mathcal{M}\left(\mathcal{G}(k) \rightarrow \bar{\nu}\left(p^{\prime}\right), \nu(p)\right)$, where $\mathcal{G}(k)$ denotes the Fourier transform of the pseudoscalar invariant $\mathcal{G}(x)$ of the electromagnetic field. Squaring the matrix element and integrating over the phase space, we obtain the production probability

$$
W=\frac{G_{\mathrm{F}}^{2} \alpha^{2}}{36(2 \pi)^{7}} \frac{m_{\nu}^{2}}{m^{4}} \int d^{4} k|\mathcal{G}(k)|^{2} k^{2}\left(1-\frac{4 m_{\nu}^{2}}{k^{2}}\right)^{1 / 2} \theta\left(1-\frac{4 m_{\nu}^{2}}{k^{2}}\right) .
$$

In order to obtain an illustrative estimate of the order of magnitude of this effect, let us simply take $\mathcal{G}(k)=\mathbf{E}_{\mathbf{0}} \cdot \mathbf{B}_{\mathbf{0}}(2 \pi)^{4} \delta^{3}(\mathbf{k}) \delta\left(\omega-2 \omega_{0}\right)$. For this field configuration, we obtain the production probability per volume and time

$$
\frac{W}{V T} \simeq 5.11\left(\frac{m_{\nu}}{1 \mathrm{eV}}\right)^{2}\left(\frac{\omega_{0}}{1 \mathrm{eV}}\right)^{2} \frac{\left(\mathbf{E}_{\mathbf{0}} \cdot \mathbf{B}_{\mathbf{0}}\right)^{2}}{B_{\mathrm{cr}}^{4}}\left(1-\frac{m_{\nu}^{2}}{\omega_{0}^{2}}\right)^{1 / 2} \theta\left(\omega_{0}-m_{\nu}\right) \mathrm{cm}^{3} \mathrm{~s},
$$

in units of $\mathrm{cm}^{3}$ and seconds. Obviously, the threshold frequency is equal to the neutrino mass, e.g., in the strong optical ultraviolet for neutrino masses at the eV scale. Equation (25) can also be interpreted as the number of pairs produced in the system and volume under consideration.

It is instructive to compare this pair-production probability with the one for $e^{+} e^{-}$ pairs: $W_{e^{+} e^{-}} \sim \int\left(E^{2}-B^{2}\right)$. Each process is triggered by a different invariant of the electromagnetic field revealing its vector or axialvector character.

Except for mass differences, the above-discussed examples are not directly sensitive to different neutrino flavors. This is different in the following example: consider a spatially constant electromagnetic vacuum field varying in time with non-vanishing $\mathbf{E} \cdot \mathbf{B} \neq 0$. Its contribution to the axial charge density is $\frac{g_{\mathrm{A}}}{e}\left\langle j_{5}^{0}\right\rangle=\frac{\alpha}{6 \pi} \frac{g_{\mathrm{A}}}{m^{2}} \frac{d}{d t} \mathbf{E} \cdot \mathbf{B}$. Therefore, such a field configuration is in complete analogy to a polarized medium. In this way, a neutrino propagating in such a field can be subject to an enhancement of flavor oscillations, similarly to a propagation in matter.

This concludes our selection of QED effective actions which govern the physics of soft electromagnetic fields and thereby connect features of the quantum vacuum with classical electrodynamics. The variety of physical effects, partly discussed here and partly still to be discovered, are manifold but share the common feature that their experimental verification will require extraordinary abilities and facilities. Nevertheless, their investigation is worthwhile and will deepen our understanding of the quantum vacuum. 


\section{Acknowledgement}

I would like to thank W. Dittrich and R. Shaisultanov for many insightful discussions and collaboration on some of the topics presented here. W. Dittrich's thoughtful reading of the manuscript is also gratefully acknowledged. Finally, it is a pleasure to thank G. Cantatore and the other organizers of this productive and enjoyable workshop for their efforts.

\section{References}

[1] W. Dittrich and H. Gies, "Probing the quantum vacuum. Perturbative effective action approach in quantum electrodynamics and its application", Springer Tracts Mod. Phys. 166, 1 (2000).

[2] H. Euler and B. Kockel, Naturwissenschaften 23, 246 (1935); W. Heisenberg and H. Euler, Z. Phys. 98, 714 (1936); V. Weisskopf, K. Dan. Vidensk. Selsk. Mat. Fy. Medd. 14, 1 (1936).

[3] J. Schwinger, Phys. Rev. 82, 664 (1951).

[4] S. L. Adler, Annals Phys. 67, 599 (1971).

[5] R. Pengo et al.; W.-T. Ni, in Frontier Tests of QED and Physics of the Vacuum, Edited by E. Zavattini, D. Bakalov and C. Rizzo, Heron Press Sofia (1998); E. Zavattini; C. Rizzo, this volume (2000).

[6] W. Dittrich and H. Gies, Phys. Rev. D58, 025004 (1998) hep-ph/9804375; Phys. Lett. B431, 420 (1998) hep-ph/9804303. W. Dittrich and H. Gies, in The Casimir Effect 50 Years Later, Proceedings of the Fourth Workshop on Quantum Field Theory under the Influence of External Conditions, Edited by M. Bordag, World Scientific, (1998) hep-ph/9903469.

[7] M. Novello, V. A. De Lorenci, J. M. Salim and R. Klippert, Phys. Rev. D61, 045001 (2000) gr-qc/9911085; S. Liberati, S. Sonego and M. Visser, quant-ph/0010055.

[8] Z. Białynicka-Birula and I. Białynicki-Birula, Phys. Rev. D 2, 2341 (1970).

[9] W. Dittrich and H. Gies, in Frontier Tests of QED and Physics of the Vacuum, Edited by E. Zavattini, D. Bakalov and C. Rizzo, Heron Press Sofia (1998) hep-ph/9806417.

[10] D. L. Burke et al., Phys. Rev. Lett. 79, 1626 (1997); A. C. Melissinos, hep-ph/9805507.

[11] P. Elmfors and B. Skagerstam, Phys. Lett. B348, 141 (1995) hep-th/9404106.

[12] H. Gies, Phys. Rev. D60, 105002 (1999) hep-ph/9812436.

[13] P. Elmfors and B. Skagerstam, Phys. Lett. B427, 197 (1998) hep-ph/9802397.

[14] H. Gies, Phys. Rev. D61, 085021 (2000) hep-ph/9909500.

[15] H. Gies, Phys. Rev. D60, 105033 (1999) hep-ph/9906303.

[16] G. Barton, Phys. Lett. B237, 559 (1990); R. Tarrach, Phys. Lett. B133, 259 (1983); M. H. Thoma, hep-ph/0005282.

[17] D. A. Dicus and W. W. Repko, Phys. Rev. Lett. 79, 569 (1997) hep-ph/9703210.

[18] A. Abada, J. Matias and R. Pittau, Phys. Rev. D59, 013008 (1999) hep-ph/9806383; Y. Aghababaie and C. P. Burgess, hep-ph/0006165. 
[19] R. Shaisultanov, Phys. Rev. Lett. 80, 1586 (1998) hep-ph/9709420.

[20] H. Gies and R. Shaisultanov, Phys. Rev. D62, 073003 (2000) hep-ph/0003144.

[21] R. Shaisultanov, hep-th/0002079.

[22] C. Schubert, Nucl. Phys. B585, 429 (2000) hep-ph/0002276.

[23] H. Gies and R. Shaisultanov, Phys. Lett. B480, 129 (2000) hep-ph/0009342.

[24] L. L. DeRaad, K. A. Milton and N. D. Hari Dass, Phys. Rev. D14, 3326 (1976); A. N. Ioannisian and G. G. Raffelt, Phys. Rev. D55, 7038 (1997) hep-ph/9612285. 